\title{
Competition Between Volatility and Overall Market Gain and the Performance of Leveraged Index Funds
}

\author{
Rainer Schad ${ }^{1}$ \\ ${ }^{1}$ MINT Center, The University of Alabama, Tuscaloosa, USA \\ Correspondence: Rainer Schad, the University of Alabama, MINT Center, Box 870209, Tuscaloosa, AL 35487, USA. \\ Tel: 1-205-348-2404.
}

Received: March 30, 2018

Accepted: April 26, 2018

Online Published: May 6, 2018

doi:10.5430/ijfr.v9n3p20

URL: https://doi.org/10.5430/ijfr.v9n3p20

\begin{abstract}
A simple yet powerful mathematical model is developed for comparison of the performance of leveraged funds versus that of their underlying index. The model strictly separates the pure market volatility from the actual long term gain of the market. This allows to study the interplay between volatility of various amplitude and gain. The fund performance is analyzed for different factors of leverage and clear limits for still acceptable market volatility are identified. For low volatility values the performance of leveraged index funds significantly exceeds the performance of what the leverage factor would suggest, reaching gains as large as 7x the gain of the index for a 3x leveraged fund. This favorable regime quickly deteriorates for intermediate values of market volatility and produces increasingly negative returns once the daily fluctuations exceed 1.5 to $2 \%$. The model provides a practical understanding of the risks and opportunities of leveraged funds.
\end{abstract}

Keywords: exchange traded funds, leveraged funds, market volatility, performance modeling

\section{Introduction}

Investment strategies involve various products of different risk levels and potential return. Traditionally, investments in stocks are considered a more risky type of investment, however with long-term favorable returns in the past. While investments in individual stocks generally require a higher level of knowledge and risk tolerance, investments in mutual funds, or more simply index funds, enjoy a high level of popularity with individual investors without the explicit need to understand the details of markets or individual stocks.

Index-related funds which potentially offer a higher rate of return are leveraged funds such as the Direxion S\&P 500 Bull \& Bear Shares with a leverage of 2x or 3x, resetting daily or monthly. A multitude of similar products is available from various investment companies. While the leverage might be obtained through various financial instruments by the investment company, the promise to the customer is, for example for the Direxion Daily S\&P 500 Bull 3x Shares, to deliver three times the daily return of the S\&P 500 index.

The simple, at first glance compelling, logic is that, if the underlying index generally goes up, the leveraged fund should climb even faster. However, warnings about their potentially disastrous long-term performance can be found at various places (Loehr, 2013; Fidelity, 2016; Carver, 2009), typically discouraging the less experienced investor from considering them. Generally, it is warned against using such funds for long-term investment strategies and rather limit their use to momentum-oriented day-trading scenarios. While this might be wise advice, it translates into saying that a leveraged fund is good as long as the market goes up, which is somewhat trivial. The Securities and Exchange Commission (SEC) has an alert (SEC, 2016) giving examples of disastrous returns of leveraged funds during periods of relatively large market volatility. A multitude of publications has analyzed the mathematics of such leveraged funds in quite some detail (Lu, 2009; Avellaneda, 2009; Trainor, 2011; Fang, 2013; Leung, 2016; Miu, 2016 and references therein). In these publications sophisticated models are used to explore the statistics of gains and risks. They yield functions describing the probability of undesirable returns. In a few publications (Trainor, 2011; Trainor, 2013; Fang, 2013) estimates of safe holding periods after which excess volatility would reverse the gains are discussed. This is in some way similar to estimating the probability of rain as, for example, a day of rain every 10 days. This would not tell whether an umbrella is needed on a specific day and it would not be safe to assume that for 9 days one could go without an umbrella. 
The purpose of this publication is to provide a simple yet exact analysis of the interplay of general market performance and overall market volatility. The data are artificially calculated for fixed values of volatility and market gain for the long-term performance of leveraged funds and serve as a basis for better risk assessment of such investment vehicles. This approach uses a strict separation of the effects of pure market volatility from the long-term gain. The performance of funds of varying level of leverage will be compared against their underlying index. The data are by no means a realistic description of actual market performance as they use fixed parameters. Therefore comparing these calculated fund performance data with actual market gyrations would be baseless as would be a comparison of the models developed in the publications referenced here with real market performance. In this sense, the results presented here do certainly not predict when to bring an umbrella. They rather describe how bad the weather is for a given set of parameters and whether it is advisable to seek shelter.

\section{Method}

The market volatility is mathematically separated from the overall market gain. The volatility is described by daily fluctuations in form of percentage gains and losses such that the underlying index remains unchanged over all time periods. The gain is described by the average daily gain in accordance with the annual performance of the index. Let $\mathrm{f}^{+}$ and $\mathrm{f}$ be the percentage fluctuation upwards and downwards, respectively, and $\mathrm{g}$ the daily gain. In the following the market will be described by alternating days up and down, corresponding to factors of $\left(1+f^{+}+g\right)$ and $\left(1-f^{-}+g\right)$. The daily gain will equal $\mathrm{g}=(\text { annual gain })^{(1 / \mathrm{N})}$, where $\mathrm{N}$ is the number of trading days in a year, typically about 252 . A $9 \%$ annual gain would result in a $0.034 \%$ (3.4 points) daily gain. The important step is that the daily fluctuation would have to be set such that up- and down-moves cancel out, regardless of the size of the fluctuations or gains. Thus they must fulfill that the combined effect of two days, one with a fluctuation up, the other with a fluctuation down will cancel out the fluctuations, leaving only the combined daily gain, or mathematically:

$$
\left(1+f^{+}+g\right) \times\left(1-f^{-}+g\right)=(1+g)^{2}
$$

Solving this for $\mathrm{f}$, we obtain

$$
f^{-}=f^{+} \times(1+g) /\left(1+f^{+}+g\right)
$$

This makes $\mathrm{f}^{+}$and $\mathrm{g}$ parameters the values of which can be set freely, while $\mathrm{f}^{-}$is calculated using Eq. (2). The performance of leveraged funds with leverage factor $\mathrm{m}$ then is calculated by multiplying $\mathrm{f}^{+}, \mathrm{f}^{-}$and $\mathrm{g}$ with $\mathrm{m}$. For example $\left(1+\mathrm{m} \times\left(\mathrm{f}^{+}+\mathrm{g}\right)\right)$ would be the gain on a day with an upwards fluctuation.

Using these equations allows to numerically calculate the performance of leveraged funds as a function of time with fluctuations and gains used as parameters. While the calculations here are based on alternating up- and down-days for the fluctuation, this specific choice has no impact on the results as factors in a multiplication are commutative.The Method section describes in detail how the study was conducted, including conceptual and operational definitions of the variables used in the study, Different types of studies will rely on different methodologies; however, a complete description of the methods used enables the reader to evaluate the appropriateness of your methods and the reliability and the validity of your results, It also permits experienced investigators to replicate the study, If your manuscript is an update of an ongoing or earlier study and the method has been published in detail elsewhere, you may refer the reader to that source and simply give a brief synopsis of the method in this section.

\section{Results}

An often presented example considers the scenario of a market that goes up a certain percentage one day and falls by that same percentage the following day. Let that percentage be $10 \%$, the underlying index would evolve by factors of ( 1 $+0.1) *(1-0.1)$, resulting in a $1 \%$ drop overall. Leveraged funds with leverage of $2 x$ or $3 x$, however, would drop by $4 \%$ and $9 \%$, respectively, much more than intuitively expected. This demonstrates that such funds bear a higher risk than one might anticipate and it also indicates that volatility in the underlying market might have detrimental effects on the performance of leveraged funds. This simple example of consecutive up- and down moves of the market resulting in a 2-day performance of $(1+x) *(1-x)$, with $x$ being the size of the daily changes simplifies to $1-x^{2}$. This explains the much higher losses of the $2 \mathrm{x}$ or $3 \mathrm{x}$ leveraged funds as those factors simply get squared. While this is instructive, the general market still trends down which obscures the effect of pure volatility. The results which now follow are calculated as described in the Methods section. They are based on a rigorous mathematical treatment of the problem which allows the exact separation of market gain from its volatility. Simplifications and assumptions and their potential impact on the reliability of the results are disclosed and discussed in the Discussion section. 


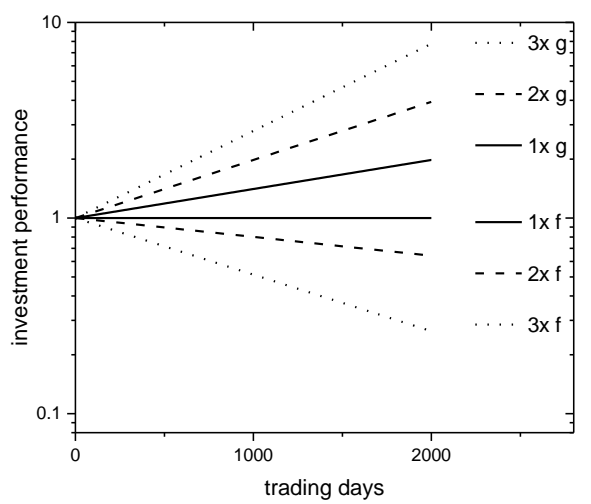

Figure 1. The performance of an index (solid lines), a 2x leveraged fund (dashed lines) and a $3 x$ leveraged fund (dotted lines) over time for either a pure annualized gain of $9 \%$ of the index (zero market volatility) - upper three lines - or pure fluctuations of $1.5 \%$ daily amplitude (and zero gain) - lower three lines

Figure 1 shows the performance of the index and $2 \mathrm{x}$ and $3 \mathrm{x}$ leveraged funds based on that same index for the two cases of pure fluctuation (1.5\% daily fluctuation) without any gain and pure gain ( $9 \%$ annual gain) without fluctuations. It is calculated over a period of 2000 trading days which approximately equals 8 years. In the case of pure fluctuations, the index remains unchanged as the factors $\mathrm{f}^{+}$and $\mathrm{f}^{-}$are designed to cancel their contributions out. The leveraged funds however, realize substantial losses over this 8 year period of about one third and three quarter for the $2 \mathrm{x}$ and $3 \mathrm{x}$ leveraged funds, respectively. Obviously, a sideways moving market is not a desirable scenario for investing in a leveraged fund. In the case of pure gain, the leveraged funds demonstrate their strength. While the underlying fund approximately doubles in the 8 year period (appreciates by $100 \%$ ), the leveraged funds increase by factors of almost 4 (a $300 \%$ increase for $2 x$ leverage) and 7.5 (a $650 \%$ increase for $3 x$ leverage). While their relative daily performance are only $200 \%$ and $300 \%$ of the underlying fund performance, the higher factors over long periods are reflective of higher order terms which essentially are a kind of compound interest. Obviously, this is a much more desirable outcome. The key issue obviously is to understand the performance of leveraged funds in the presence of both fluctuations and a steady gain.

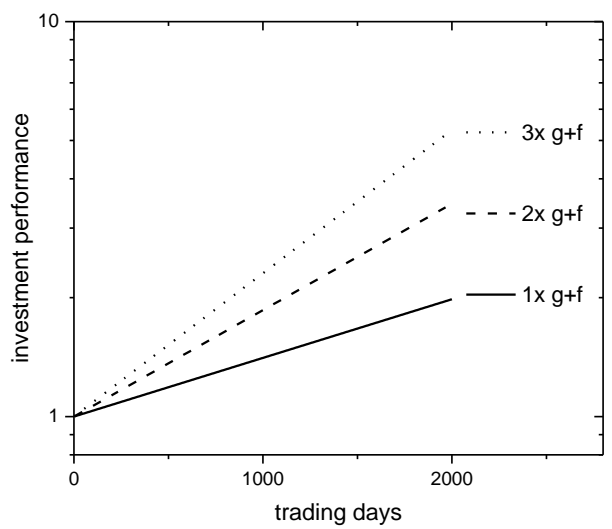

Figure 2. The performance of an index (solid line), a $2 x$ leveraged fund (dashed line) and a $3 x$ leveraged fund (dotted line) over time for an annualized gain of $9 \%$ for the index combined with market volatility of $0.8 \%$ daily fluctuations 
An example is given in figure 2, for an annualized gain of $9 \%$ and fluctuations of $0.8 \%$. The latter value is typical for the last few years 2010 to 2015 (Easterling, 2016), a relatively calm period. The performance of the index is identical to that shown in figure 1 in the absence of fluctuations, gaining a factor of 2 over that 8 year period. The yields of the leveraged funds are diminished due to the added fluctuations. The $2 \mathrm{x}$ leveraged fund increases by a factor of 3.4 (a $240 \%$ increase) and the $3 x$ leveraged fund increases by a factor of 5 (a $400 \%$ increase). These data still look attractive and for both leverage factors lie above the daily leverage advantage. The question is, from which point on the detrimental effect of fluctuations will reverse the amplified gain.

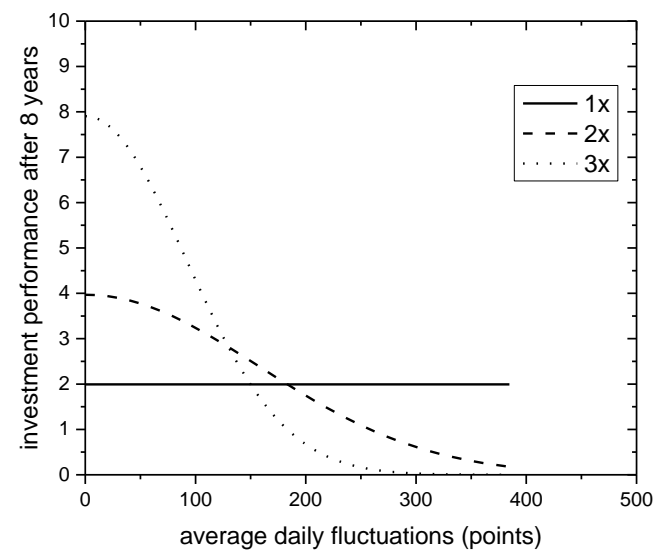

Figure 3. The long-term performance of an index (solid line), a $2 x$ leveraged fund (dashed line) and a $3 x$ leveraged fund (dotted line) over a 8 year period with an annualized gain of $9 \%$ of the index as a function of the amplitude of market fluctuations

In order to get a sense of this negative impact of the size of fluctuations, the data were analyzed for a fixed time period ( 8 years) as a function of the fluctuation amplitude, as shown in figure 3. As shown before, for small fluctuations the leveraged funds yield a strongly amplified return compared to the index. Actually the low-fluctuation return by far exceeds the leverage factor. This advantage quickly diminishes as the fluctuation amplitude increases. At about $1.3 \%$ daily fluctuations the $2 \mathrm{x}$ and $3 \mathrm{x}$ leveraged funds show equal performance, but still with a $180 \%$ gain compared to the index with an unchanged $100 \%$ gain. Beyond $1.5 \%$ fluctuations the $3 x$ leveraged fund underperforms the index and at about $1.8 \%$ fluctuations also the $2 \mathrm{x}$ leveraged fund drops below the index performance. At that point the return of the $3 x$ leveraged fund actually turns negative and at $2.6 \%$ also the $2 x$ leveraged fund performance becomes negative, while the index, of course, still yields its $100 \%$ gain. A prolonged period of $3 \%$ fluctuations practically wipes out the investment in a $3 x$ leveraged fund, despite the steady overall gain of the index. This is in agreement with the examples given in (SEC, 2016) which mostly refer to the period of large fluctuations during the strong downturn of markets between 2007 and 2009. Interestingly, these fluctuation levels at which the leveraged fund performances cross the index performance are independent of the holding period, thus the intersections of the lines in figure 3 would be the same to a 10 day holding period. What does vary with the duration of the holding period is the steepness of the functions in figure 3 resulting in exceedingly larger advantages at low fluctuation levels or disadvantages at higher fluctuation levels with longer holding periods.

In the following figure 4 these crossing points are plotted as a function of leverage multiple to give an idea how fluctuation tolerant various levels of leverage are. The solid line, for instance indicates the fluctuation level at which the leveraged fund equals its nominat performance multiple, like a $3 x$ leveraged fund yielding 3 times the return of the index. Below this line it performes better than nominal, above it underperforms. The dashed and the dotted lines indicate the fluctuation levels at which the performance of a leveraged fund only equals that of the $2 x$ leveraged fund or the unleveraged index, respectively. The graph is extended to include leverage levels not available on the market to give insight how such inverstments might perform. A $5 \mathrm{x}$ leveraged fund would become unattractive at fluctuation levels of about $1 \%$. 


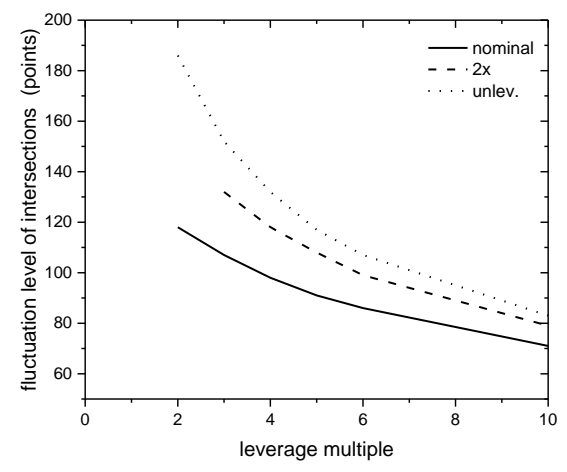

Figure 4. The fluctuation level $g$ at which the performance of a leveraged fund matches the nominal performance (solid line), the performance of a $2 x$ leveraged fund (dashed line), or the performance of the unleveraged index (dotted line) as a function of leverage multiple

\section{Discussion}

Leveraged funds are indeed an excellent long-term inverstment with time-horizons of years. Besides confidence in the general market development, the investor however needs to have an idea about to be expected level of volatility. As one might expect, the level of fluctuations will depend on various factors. Some of those will be inherent to the composition of the index, like breadth of the index (more companies included in the index will smoothen out individual stocks' variations), the size of the companies, and the maturity of the sector (for example consumer staples vs. biotechnology) and the economy (developed vs. developing markets). In addition, the level of volatility will vary with the level of (perceived) economic distress. Certainly, a $3 \mathrm{x}$ leveraged fund based on the performance of 10 biotechnology startup companies would not be a sound investment. However, a broad index based on large companies in a developed market like the S\&P 500 typically has multiyear periods of sub 1\% average fluctuations (Easterling, 2016) interrupted by sometimes brief (1987) spikes in volatility or longer high-volatility periods, for instance during the build and bust of the .com-bubble (1998 to 2003).

The appropriateness of leveraged index funds as a long-term investment strategy, as usual in investing, is best assessed in hindsight but harder to predict for future use. Over the last couple of years (2010 to 2015) of relatively modest fluctuations and strong gains in US markets, leveraged funds have built an impressive track record. In periods of low fluctuations the aggregate gain can be much higher than the daily leverage factor suggests, in perfect agreement with the results found here. This advantage is quickly lost with increasing fluctuations, most pronounced for higher levels of leverage. While many of the more cautious assessments (Carver, 2009; SEC, 2016; Avellaneda, 2009) of leveraged funds are based on data including the high-volatility era of 2008-2009, more recent publications (Trainor, 2011; Trainor, 2013) shed a more favorable light on that matter.

In publication (Trainor, 2013) a similar study is presented, analyzing the interplay between volatility and return of leveraged funds with recommendations for maximum holding periods before a certain level of underperformance (called decay) is reached. This paper actually holds more results than the authors discuss. They show that for certain parameters, like $3 \times$ leverage and $20 \%$ volatility a decay of $2 \%$ is reached after 42 trading days, which would be the acceptable holding period. Their data however also demonstrate that holding that fund for a longer period would bring the decay back to the $2 \%$ level after about 140 trading days and would turn into a positive decay (actually an over-performance) after 180 trading days. This surprising observation is at first glance different from what is observed here and might be due to the different mathematical model used in (Trainor, 2013).

The model developed here is straightforward and transparent, yet delivers powerful insight into the performance and limitations of leveraged funds keeping the effects of fluctuations strictly separated from the overall market gain. Although the calculations are performed for alternating up and down fluctuations, the same results would be obtained for longer bear markets (many accumulated down steps) followed by a complementary recovery, as long as the overall long-term gain still exists. This holds true simply because multiplication of factors is commutative and thus their order is irrelevant for the outcome. This model does not indicate any maximum holding periods. For a given level of volatility the useful holding period might actually be infinite and indeed, over the last several years certain leveraged funds have performed very well. For example, the S\&P500 has gained about 70\% over the last 3 years while the 
corresponding $3 \mathrm{x}$ leveraged fund did quadruple. This period was characterized by an overall low level of volatility interrupted by only short outbreaks of higher volatility levels. The practical problem lies in the variations of volatility which might be as hard to predict as the overall market trend. The calculations presented here do not possess any predictive power nor is its framework of rigid parameters suitable to model actual market data, yet they provide a solid base to assess acceptable values of volatility in dependence of leverage factor.

Not included in this study where inverse funds, leveraged or not, which are similar in the mathematics but distincly different in some critical aspects. First, as shown in (Trainor, 2013), they are inherently more susceptible to decay than their positively leveraged counterparts. Second, most markets have a built-in or assumed tendency to go up over the long term, either through economic growth, depletion of a commodity or simply inflation. Thus the useful holding period for inverse funds must generally be limited to those periods over which the underlying market has a negative trajectory. The duration and amplitude of such reverse periods can always be clearly observed and modeled in hindsight but are inherently more difficult to predict. In addition, downwards markets have typically a higher level of volatility which, of course, would also be detrimental for inverse leveraged funds. Thus inverse funds, and in particular those with leverage, are useful only for investors with a good predictive capability of future market direction and an intraday time horizon or with additional predictive capabilities of market fluctuations when using longer inverstment periods.

Still the model presented here has some limitations or oversimplifications. A few factors are not considered here, like the fund administrative fees and commissions. Those will somewhat reduce the fund performance but not change the picture in a qualitative way. Probably more important will be tracking errors (Holzhauer, 2013; Loviscek, 2014), the degree to which the fund delivers its promised daily leverage factor which here is assumed to be accurate. While occasional and random deviations might not have much of an effect, systematic under performance on upswings combined with over performance on down-swings would have a significant impact in the long term. It also will be worth analyzing in how far the results obtained here, using a constant average fluctuation amplitude, will change when allowing a variation of the fluctuation amplitude with the same average.

\section{References}

Avellaneda, M., \& Stanley, J. Z. (2009). Path-Dependence of Leveraged ETF Returns. Retrieved from SSRN: http://ssrn.com/abstract=1404708 or http://dx.doi.org/10.2139/ssrn.1404708

Carver, A. B. (2009). Do Leveraged and Inverse ETFs Converge to Zero?. ETFs and Indexing Exchange Traded Funds, 2009(1), 144-149.

Easterling (Ed.). (2016). Crestmont Research, Volatility in Perspective. Retrieved March 6, 2016, from http://www.crestmontresearch.com/docs/Stock-Volatility-Perspective.pdf

Fang, M., \& Perng, C.-T. (2014). A study of leveraged ETF based on geometric return. Applicable Analysis, 93(9), 1884-1894. https://doi.org/10.1080/00036811.2013.851863

Fidelity. (2016). Types of ETFs/ETPs. Retrieved March 20, 2016, from https://www.fidelity.com/learning-center/investment-products/etf/types-of-etfs/overview

Holzhauer, H. M., Lu, X., McLeod R. W., \& Mehran, J. (2013). Bad news bears. Managerial Finance, 39(12), 1169-1187. https://doi.org/10.1108/MF-09-2012-0203

Leung, T., \& Santoli, M. (2016). Leveraged Exchange-Traded Funds.

Loehr, R., \& Lamb, R. (2013). Long-Term Investing With Leveraged Exchange Traded Funds. International Journal of Arts and Commerce, 2(4), 27-34.

Loviscek, A., Tang, H., \& Xu, X. E. (2014). Do leveraged exchange-traded products deliver their stated multiples?, Journal of Banking \& Finance, 43, 29-47. https://doi.org/10.1016/j.jbankfin.2014.02.008

Lu, L., Wang, J., \& Zhang, G. (2009). Long Term Performance of Leveraged ETFs. Retrieved from SSRN: http://ssrn.com/abstract=1344133 or http://dx.doi.org/10.2139/ssrn.1344133

Miu, P., \& Charupat, N. (2016). Leveraged Exchange-Traded Funds. Palgrave Macmillan US.

SEC. (2016). US Securities and Exchange Commission, Leveraged and Inverse ETFs: Specialized Products with Extra Risks for Buy-and-Hold Investors. Retrieved March 23, 2016, from http://www.sec.gov/investor/pubs/leveragedetfs-alert.htm

Trainor, W. J. Jr. (2011). Solving the Leveraged ETF Compounding Problem. The Journal of Index Investing Spring, $1(4), 66-74$.

Trainor, W. J. Jr., \& Carroll, M. G. (2013). Forecasting Holding Periods for Leveraged ETFs Using Decay Thresholds: Theory and Applications. Journal of Financial Studies \& Research, 1-12. 